\title{
Reconstruction of the Foot Using a Superficial Inferior Epigastric Artery Free Flap
}

\author{
Jin Ho Han, Hyun Woo Shin, Kun Chul Yoon, June-Kyu Kim \\ Department of Plastic and Reconstructive Surgery, Kangbuk Samsung Hospital, Sungkyunkwan University School of Medicine, Seoul, Korea
}

\begin{abstract}
When foot reconstruction is performed in the pretibial area, the ankle, or the dorsum of the foot, the need for a reliable flap remains a challenge. We found that the superficial inferior epigastric artery (SIEA) free flap can be used as an alternative tool for this purpose, as it helps to solve the problems associated with other flaps. We describe 2 cases in which we reconstructed the foot using an SIEA free flap, which was pliable enough to fit the contours of the area. Postoperatively, the flaps were intact and showed excellent aesthetic results. Thus, the SIEA free flap can be an alternative tool for patients with a low body mass index who undergo reconstructive surgery involving the pretibial area, ankle, knee, or dorsum of the foot, all of which require a soft and flexible flap.
\end{abstract}

Keywords Free tissue flaps / Lower extremity / Microsurgery
Correspondence: Hyun Woo Shin Department of Plastic and Reconstructive Surgery, Kangbuk Samsung Hospital, Sungkyunkwan University School of Medicine, 29, Saemunan-ro, Jongno-gu, Seoul 03181, Korea

Tel: +82-2-2001-2178

Fax: +82-2-2001-2177

E-mail:mdshin7@naver.com

This article was presented as an e-poster at the 74th Congress of the Korean Society of Plastic and Reconstructive Surgeons on November 17-20, 2016 in Seoul, Korea.

\section{INTRODUCTION}

When reconstruction of the foot is performed in the pretibial area, ankle, or the dorsum of the foot, the need for a reliable flap remains a challenge.

The anterolateral thigh free flap and the superficial circumflex iliac artery perforator (SCIP) free flap are popular methods used in reconstructive surgery in these regions. However, intramuscular dissection is more time-consuming and requires meticulous dissection under high magnification [1], and poses the risk of injury to the pedicle during the thinning of the flap.

Although the SCIP free flap is pliable and soft, the relatively short length of the pedicle (mean pedicle length, $4.9 \mathrm{~cm}$ ) [2] and the fact that it includes small-caliber vessels of less than 1.0 $\mathrm{mm}$ [3] can cause difficulties for the surgeon while performing the micro-anastomosis procedure. We verified that the use of an superficial inferior epigastric artery (SIEA) free flap helped to solve these problems while producing excellent results.

\section{CASES}

\section{Case 1}

A 62-year-old man who was referred to our hospital presented with an ulcer on his right foot that began after callus removal. The ulcer did not heal and gradually developed necrosis. He went to a local clinic where he was diagnosed with diabetes mellitus and was started on medical therapy and wound management for a month.

When he visited our clinic, signs of infection were observed, with a foul odor at the wound site; we therefore prescribed treatment with empirical antibiotics. Furthermore, his serum hemoglobin A1c level was 10.2\%, and he had decreased sensa- 


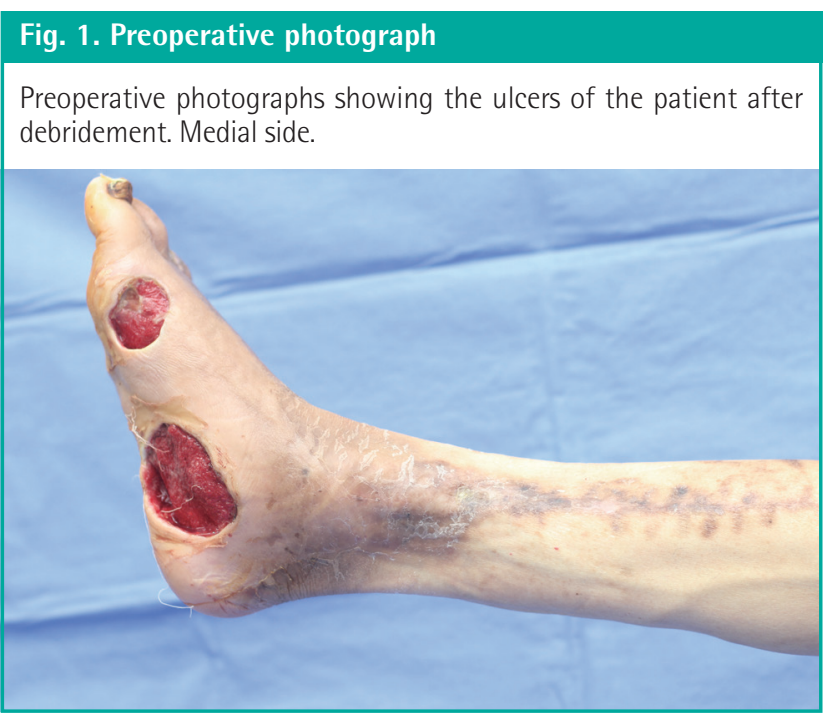

Fig. 2. Intraoperative photograph

Intraoperative photograph showing the flap donor site in the abdomen, after superficial inferior epigastric artery flap elevation.

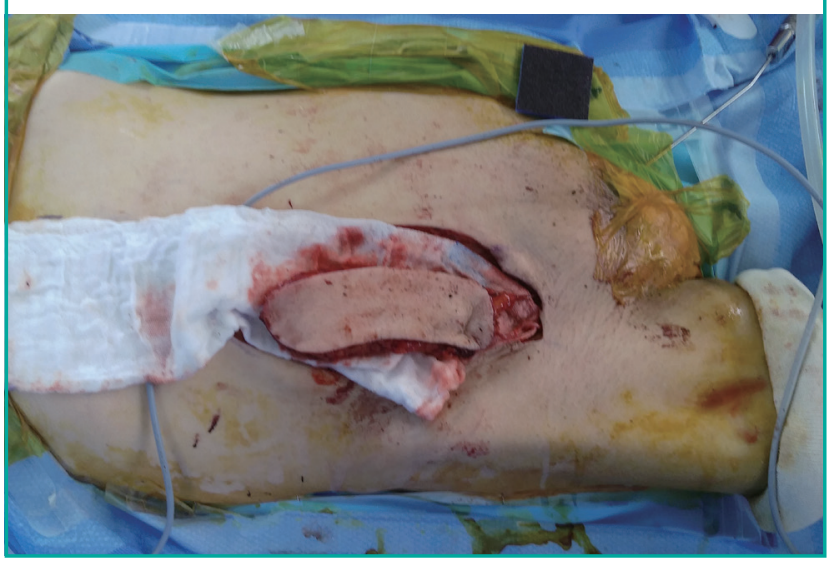

tion in his right foot. Angiography performed by a radiologist found partial occlusion in the anterior tibial artery and the peroneal artery.

He was admitted to the plastic surgery unit and he underwent wound debridement twice. The ulcer was found to extend to the underlying muscles, and the defect measured $15 \mathrm{~cm} \times 6 \mathrm{~cm}$ (Fig. 1). Once the signs of infection subsided, we reconstructed the area with an SIEA free flap (Fig. 2). The patient was lean, and had a body mass index (BMI) of $17 \mathrm{~kg} / \mathrm{m}^{2}$, which made him a suitable candidate for reconstruction with an SIEA free flap. The flap was pliable enough to cover the defect site, and it fit the contours of the foot well. After adequate preparation of the vessels, a 7-cm pedicle was created (Figs. 3, 4), and microsurgical anastomoses were performed at the level of the ankle using the posterior tibial artery with end-to-side anastomosis and a vein with end-to-end anastomosis using 10-0 nylon sutures.

\section{Fig. 3. Intraoperative photograph}

Intraoperative photograph of the superficial inferior epigastric artery free flap after detachment from the donor site (pedicle length, approximately $7 \mathrm{~cm}$ ).

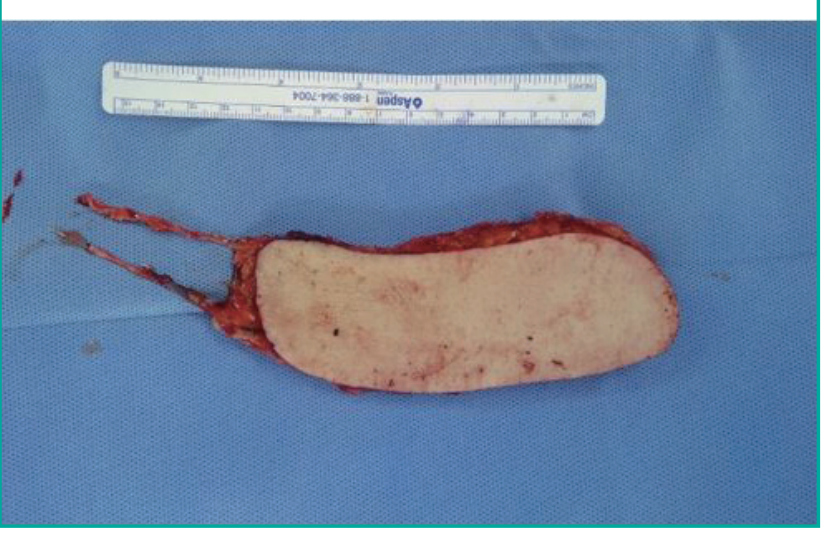

Fig. 4. Intraoperative photograph

Primary closure of the flap donor site.

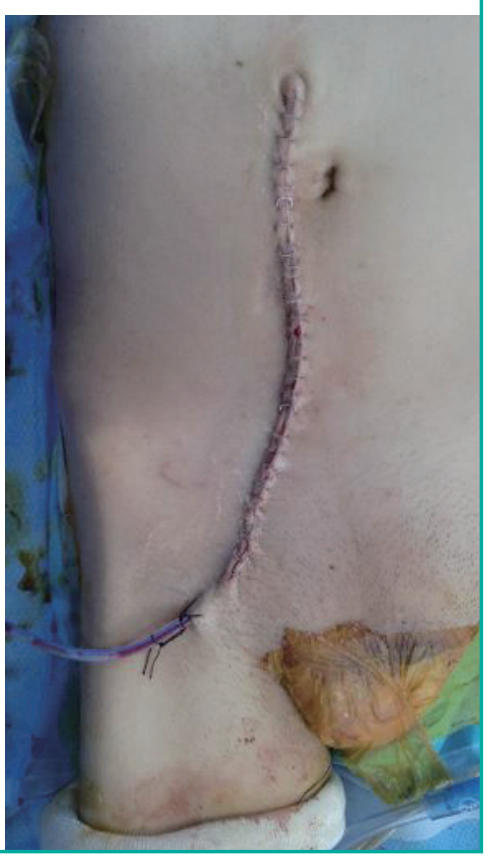

Postoperatively, the patient's serum glucose levels were controlled aggressively, and flap engraftment was performed successfully. On postoperative day (POD) 27, the sutures were removed and the patient was discharged. At follow-up 2 months later, the flap was intact and it showed excellent aesthetic results (Fig. 5).

\section{Case 2}

A 45-year-old woman presented with a soft tissue defect over the right medial malleolus. She had previously suffered tibiofibular fractures caused by a road traffic accident and had undergone open reduction and internal fixation. At the time of pre- 


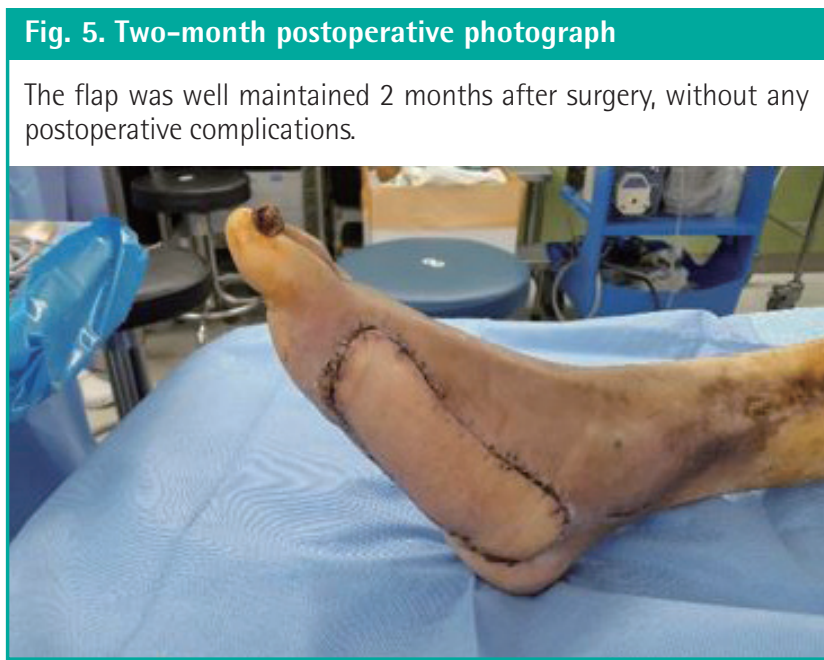

Fig. 6. Preoperative photograph

Preoperative photograph showing the soft tissue defect and the underlying hardware in the patient.

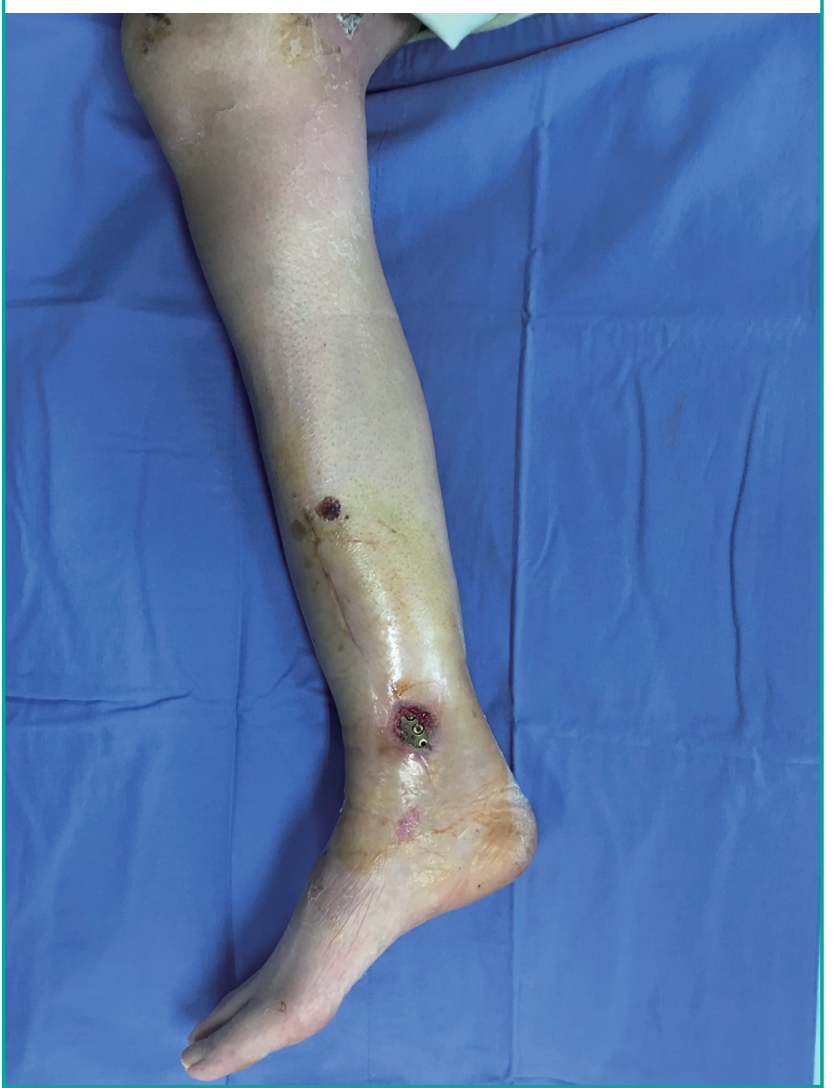

sentation, the soft tissue defect measured $6 \mathrm{~cm} \times 4 \mathrm{~cm}$, and the underlying hardware was exposed (Fig. 6). She was transferred to the plastic surgery department for reconstruction of the soft tissue defect over the right medial malleolus. The patient had a BMI of $20 \mathrm{~kg} / \mathrm{m}^{2}$. We marked the SIEA with the help of a Doppler ultrasound, around the midpoint of the line connecting the

\section{Fig. 7. Intraoperative photograph}

Intraoperative photograph of the superficial inferior epigastric artery free flap from the lower abdominal area.

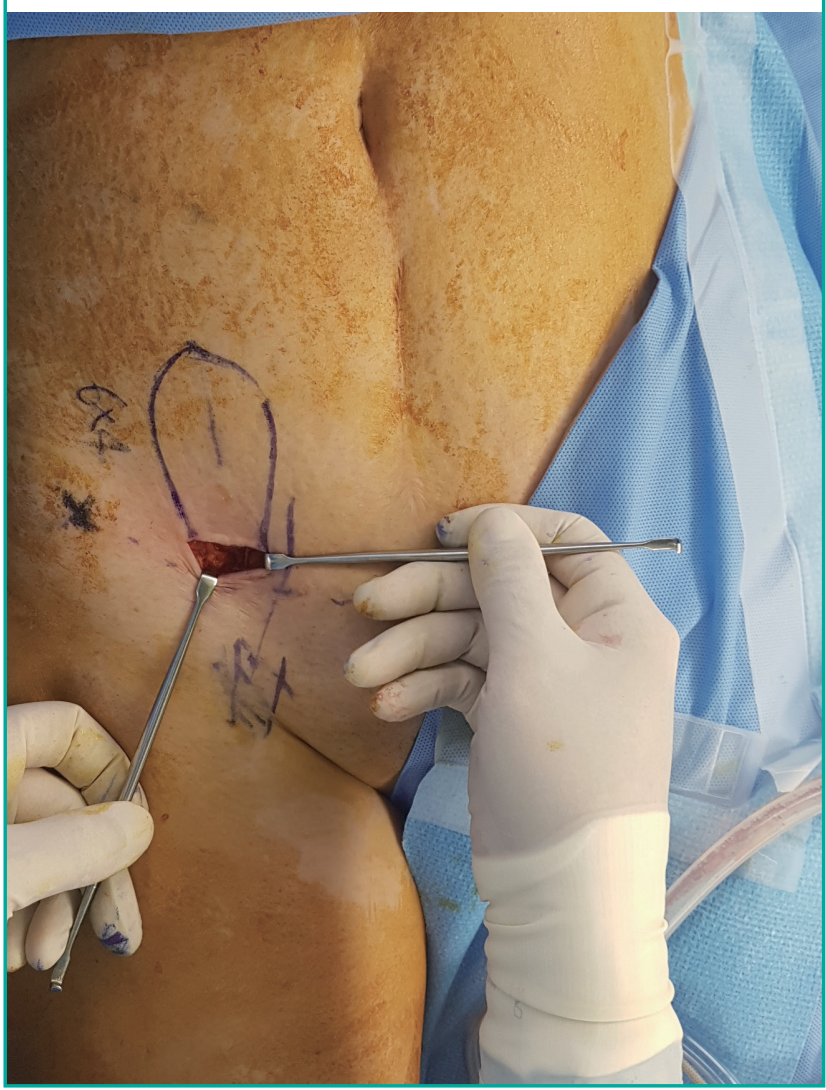

anterior superior iliac spine and the pubic tubercle, and then carried out a dissection to expose the superficial inferior epigastric vessels.

Once we marked the design of the flap to fit the measured defect, we dissected until the fascia of Scarpa was revealed (Fig. 7), and elevated the flap above the fascia.

The harvested SIEA flap was fixed over the defect site, and the posterior tibial vessels were anastomosed with the superficial inferior epigastric vessels in an end-to-side manner. The sutures were removed on POD 17 and the reconstructed site showed relatively good contours (Fig. 8) when compared to the uninjured side. The flap was intact and there were no complications.

\section{DISCUSSION}

In 1863, Wood reconstructed a forearm defect using an SIEA free flap with preserved donor site muscle and fascia. In 1971, Antia and Buch [4] used an SIEA free flap in reconstructive surgery of the face for the first time.

In 1992, Stern and Nahai [5] described 27 cases of reconstructive surgery of the face and limbs that used SIEA free flaps. The 


\section{Fig 8. One-month postoperative photograph}

The flap was well maintained 1 month after surgery. No postoperative complications were observed.

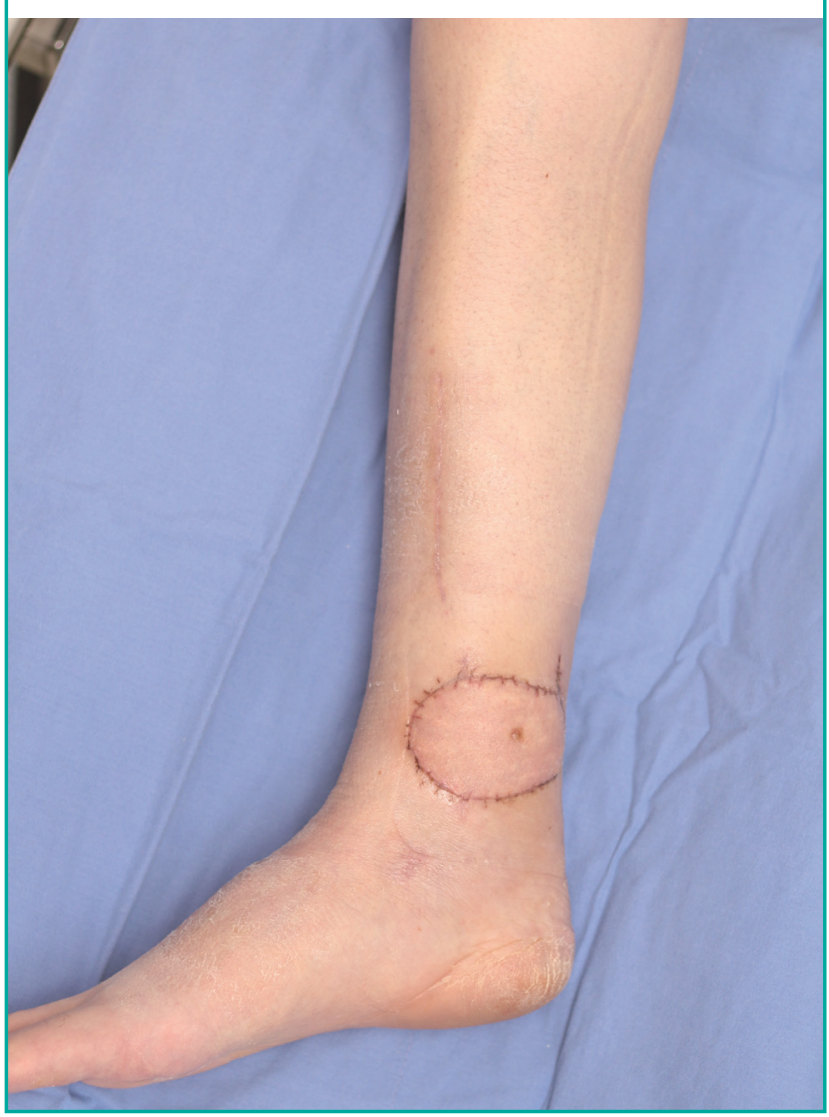

advantage of this flap is that the vessels exit the flap from one of its edges, which makes it easy to use in the reconstruction of the face or limbs.

In 2008, Nasir and Aydin [6] reported the advantages of an SIEA free flap in the reconstruction of the extremities. SIEA free flaps were found to cover extensive defects adequately, and the healing of the donor sites was cosmetically excellent. Furthermore, if necessary, debulking surgery could be performed under local anesthesia.

The lower extremities are away from the central circulation and have a high incidence of vascular complications after reconstructive surgery. Moreover, a large flap is required for the reconstruction of the lower extremities due to the poor laxity of the local tissue [6].

During the movements of the lower limbs, their surface contours change, meaning that a flap that does not have contours matching the rest of the limb will not only be aesthetically unpleasant but will also be of poor functional utility. Hence, fasciocutaneous flaps are preferred over muscle flaps when reconstructing the lower limbs.
The SIEA free flap allows a larger flap than does the SCIP free flap. Therefore, an SIEA free flap is suitable for the reconstruction of the lower limbs, as various sizes of flaps may be required.

In patients with diabetes mellitus with peripheral vascular insufficiency, it is important to ensure that the flap has a long enough pedicle to perform the anastomosis of the vessels of the flap with healthy recipient vessels, which may be far from the site of the flap [7].

The physical properties of the SIEA free flap are similar to those of the SCIP free flap, and when the skin flap is harvested, the muscle layer at the donor site is not damaged [3]. Therefore, SIEA flaps have excellent results in terms of healing at the donor site [8]

Additionally, an SIEA flap can provide a pedicle that is $2-3 \mathrm{~cm}$ longer than is provided by the SCIP free flap, and has a larger mean caliber of vessels $(1.9 \mathrm{~mm})$ [9], which makes reconstruction of the lower extremity easier when performing microsurgery.

In the case of a perforator flap, such as the SIEA free flap, dissection of the perforator vessels requires fine microsurgical skills and a thorough understanding of the anatomic variations of the flap pedicle.

The lower abdomen exhibits a layered structure known as the fascia of Scarpa, which allows relatively accurate and safe flap elevation.

However, in obese patients, it is difficult to make a thin flap. Therefore, when selecting patients for reconstruction using the SIEA free flap method, the thickness of the skin in the lower abdominal area should be evaluated preoperatively, and patients who have a low BMI are better candidates for this procedure.

It should be noted that the SIEA has a small caliber and does not exist in $25 \%$ of Korean women. Therefore, careful evaluation of the lower abdominal vasculature and screening are important for this reconstructive procedure to be successful [10].

In our cases, after SIEA flap elevation, the donor site was closed vertically. However, from a cosmetic point of view, a bikini incision (abdominoplasty) may be used with excellent cosmetic results [11].

The SIEA free flap can be used as an alternative tool for the reconstruction of lower extremity defects when a soft, pliable, and large flap is required.

\section{CONFLICT OF INTEREST}

No potential conflict of interest relevant to this article was reported. 


\section{PATIENT CONSENT}

The patient provided written informed consent for the publication and the use of their images.

\section{REFERENCES}

1. Yu P. Characteristics of the anterolateral thigh flap in a Western population and its application in head and neck reconstruction. Head Neck 2004;26:759-69.

2. Choi DH, Goh T, Cho JY, et al. Thin superficial circumflex iliac artery perforator flap and supermicrosurgery technique for face reconstruction. J Craniofac Surg 2014;25:2130-3.

3. Koshima I, Nanba Y, Tsutsui T, et al. Superficial circumflex iliac artery perforator flap for reconstruction of limb defects. Plast Reconstr Surg 2004;113:233-40.

4. Antia NH, Buch VI. Transfer of an abdominal dermo-fat graft by direct anastomosis of blood vessels. Br J Plast Surg 1971;24:15-9.
5. Stern HS, Nahai F. The versatile superficial inferior epigastric artery free flap. Br J Plast Surg 1992;45:270-4.

6. Nasir S, Aydin MA. Reconstruction of soft tissue defect of lower extremity with free SCIA/SIEA flap. Ann Plast Surg 2008;61:622-6.

7. Park JE, Rodriguez ED, Bluebond-Langer R, et al. The anterolateral thigh flap is highly effective for reconstruction of complex lower extremity trauma. J Trauma 2007;62:162-5.

8. Healy C, Ramakrishnan V. Autologous microvascular breast reconstruction. Arch Plast Surg 2013;40:3-10.

9. Reardon CM, O'Ceallaigh S, O'Sullivan ST. An anatomical study of the superficial inferior epigastric vessels in humans. Br J Plast Surg 2004;57:515-9.

10. Kim BJ, Choi JH, Kim TH, et al. The superficial inferior epigastric artery flap and its relevant vascular anatomy in korean women. Arch Plast Surg 2014;41:702-8.

11. Nasir S, Aydin MA. Upper extremity reconstruction using free SCIA/SIEA flap. Microsurgery 2010;30:37-42. 